ARTICLE

https://doi.org/10.1038/s41467-020-14521-8

\title{
Strain-driven growth of ultra-long two-dimensional nano-channels
}

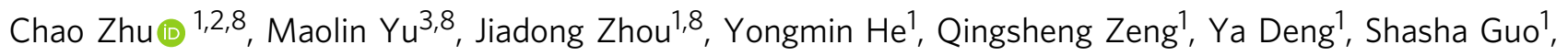
Mingquan $\mathrm{Xu}^{2}$, Jinan Shi ${ }^{2}$, Wu Zhou ${ }^{2}$, Litao Sun (1) ${ }^{4}$, Lin Wang $\mathbb{D}^{5}$, Zhili Hu${ }^{3}$, Zhuhua Zhang ${ }^{3 \star}$, Wanlin Guo (i) ${ }^{3 \star} \&$ Zheng Liu (1) $1,6,7 \star$

Lateral heterostructures of two-dimensional transition metal dichalcogenides (TMDs) have offered great opportunities in the engineering of monolayer electronics, catalysis and optoelectronics. To explore the full potential of these materials, developing methods to precisely control the spatial scale of the heterostructure region is crucial. Here, we report the synthesis of ultra-long $\mathrm{MoS}_{2}$ nano-channels with several micrometer length and 2-30 nanometer width within the $\mathrm{MoSe}_{2}$ monolayers, based on intrinsic grain boundaries (GBs). Firstprinciples calculations disclose that the strain fields near the GBs not only lead to the preferred substitution of selenium by sulfur but also drive coherent extension of the $\mathrm{MoS}_{2}$ channel from the GBs. Such a strain-driven synthesis mechanism is further shown applicable to other topological defects. We also demonstrate that the spontaneous strain of $\mathrm{MoS}_{2}$ nanochannels can further improve the hydrogen production activity of GBs, paving the way for designing GB based high-efficient TMDs in the catalytic application.

\footnotetext{
${ }^{1}$ School of Materials Science and Engineering, Nanyang Technological University, Singapore 639798, Singapore. ${ }^{2}$ School of Physical Sciences, CAS Key Laboratory of Vacuum Physics, University of Chinese Academy of Sciences, Beijing 100049, China. ${ }^{3}$ State Key Laboratory of Mechanics and Control of Mechanical Structures, Key Laboratory for Intelligent Nano Materials and Devices of Ministry of Education, and Institute of Nanoscience, Nanjing University of Aeronautics and Astronautics, Nanjing 210016, China. ${ }^{4}$ SEU-FEI Nano-Pico Center, Key Laboratory of MEMS of Ministry of Education, Collaborative Innovation Center for Micro/Nano Fabrication, Device and System, Southeast University, Nanjing 210096, People's Republic of China. ${ }^{5}$ Key Laboratory of Flexible Electronics \& Institute of Advanced Materials, Jiangsu National Synergetic Innovation Center for Advanced Material, Nanjing Tech University, 30 South Puzhu Road, Nanjing 211816, China. ${ }^{6}$ Environmental Chemistry and Materials Centre, Nanyang Environment and Water Research Institute, Singapore, Singapore. ${ }^{7}$ CINTRA CNRS/NTU/THALES, UMI 3288, Research Techno Plaza, 50 Nanyang Drive, Border X Block, Level 6, Singapore 637553, Singapore. ${ }^{8}$ These authors contributed equally: Chao Zhu, Maolin Yu, Jiadong Zhou. *email: chuwazhang@nuaa.edu.cn; wlguo@nuaa.edu.cn; z.liu@ntu.edu.sg
} 
T ransition metal dichalcogenides (TMDs) lateral heterostructures have shown promising applications in modern semiconductor devices due to their unique electronic and optical properties ${ }^{1-6}$ because their components are spatially separated and the band offset is tailorable ${ }^{7}$. However, achieving this goal requires precise control of growth not only in lattice matching but also in material dimension, considering that the Schottky barrier height, band offset, and bandgap depend vitally on the component interfacial structure and domain size ${ }^{8,9}$. The domain size should be within tens of nanometers or even several nanometers, which is essential for band adjustment and quantum confinement. By far, epitaxial growth has been now most widely adapted to design various lateral heterostructures, including $\mathrm{MoS}_{2}-\mathrm{MoSe}_{2}{ }^{10}, \mathrm{MoS}_{2}-\mathrm{WS}_{2}{ }^{11,12}, \mathrm{MoSe}_{2}-\mathrm{WSe}_{2}{ }^{13,14}$, and etc. Although atomically sharp heterointerface has been demonstrated $^{15,16}$, the fabrication of nanoscale lateral heterostructures is still a great challenging because of the fast growth rate of TMDs $s^{17-19}$ and the difficulties of quantitative control of vapor sources. Therefore, other growth strategies are needed to create narrow heterostructures.

Defects, mainly dislocations and grain boundaries (GBs), exist extensively in two-dimensional crystals. The previous understandings of defects focus mostly on the analysis of atom arrangement and investigation of various properties ${ }^{20-24}$. Until recently, dislocations at heterointerface are found to act as catalysts to guide the growth of one-dimensional (1D) TMD nanochannels, making point defects a character for the growth of TMD in-plane heterostructure ${ }^{25}$. Unfortunately, the length of these nano-channels $(<100 \mathrm{~nm})$ is incomparable with the matrix flakes (usually several to tens of microns), which may restrict their practical applications. In contrast to dislocations, the microscale length and atomic-level width of 1D defects make GBs become more potential frameworks for the possible growth of flake-sized narrow heterostructure domains.

Here, using intrinsic $60^{\circ} 1 \mathrm{D} \mathrm{GBs}$ as the catalyst, we report the growth of ultra-long $\mathrm{MoS}_{2}$ nano-channels embedded in $\mathrm{MoSe}_{2}$ monolayer matrix, as shown in Fig. 1. The $\mathrm{MoS}_{2}$ channels, possessing atomically sharp heterointerface with $\mathrm{MoSe}_{2}$ host, can reach to several micrometers long while keeping a flexible width from 2 to $30 \mathrm{~nm}$. The theoretically proposed strain-driven growth mechanism based on density functional theory (DFT) calculations perfectly fits into the experimental observations and maps of strain distribution.

\section{Results}

$\mathrm{MoS}_{2}$ channel embedded in $\mathrm{MoSe}_{2}$ monolayer. Figure 2a presents an optical image of the $\mathrm{MoSe}_{2}-\mathrm{MoS}_{2}$ flake, which appears to be a six-point star (light purple contrast). The formation mechanism of the star shape is due to the collision of three growing $\mathrm{MoSe}_{2}$ domains with a concurrently growing larger triangle, as illustrated by phase-field simulation shown in Fig. $2 \mathrm{c}$ and Supplementary Movie 1. The simulation suggests that six GBs are starting from the star center to its six bottom concave points. Fast Fourier transformation (Fig. 2d) of the heterointerface region shows the separated $\mathrm{MoS}_{2}$ and $\mathrm{MoSe}_{2}$ (110) spots with d-spacing of 0.164 and $0.158 \mathrm{~nm}$, respectively. The inset in Fig. 2d shows the corresponding annular dark-field scanning transmission electron microscope (ADF-STEM) image of the $\mathrm{MoSe}_{2}-\mathrm{MoS}_{2}$ heterostructure. A typical straight $\mathrm{MoS}_{2}$ channel is shown in Fig. 2e. It is $\sim 20 \mathrm{~nm}$ in width and at least $600 \mathrm{~nm}$ in length because we cannot see the entire channel due to the limitation of $1.2 \mu \mathrm{m}$ holes on the gold supported foil. The channel has lower brightness than $\mathrm{MoSe}_{2}$ host matrix on account of the atomic number contrast for ADF-STEM images. Such straight channels usually start at the vertexes of obtuse angles between two corners and extend toward the inside direction, as can be seen in the scanning electron microscopy (SEM) image of Fig. $2 b$ and Supplementary Fig. 1, indicating the ultra-long feature of channels (up to tens of micrometers depending on the scale of matrix flakes). High-resolution ADF-STEM image illustrates that the straight channel grows along the zigzag direction of the hexagon lattice and possess atomically sharp sidewalls coherently bonding to $\mathrm{MoSe}_{2}$ monolayer (Fig. 2f, g).

Atomic structure analysis of $\mathrm{MoS}_{2}$ channel. Further investigation of the atomic structure of the rectangular area in Fig. 3a

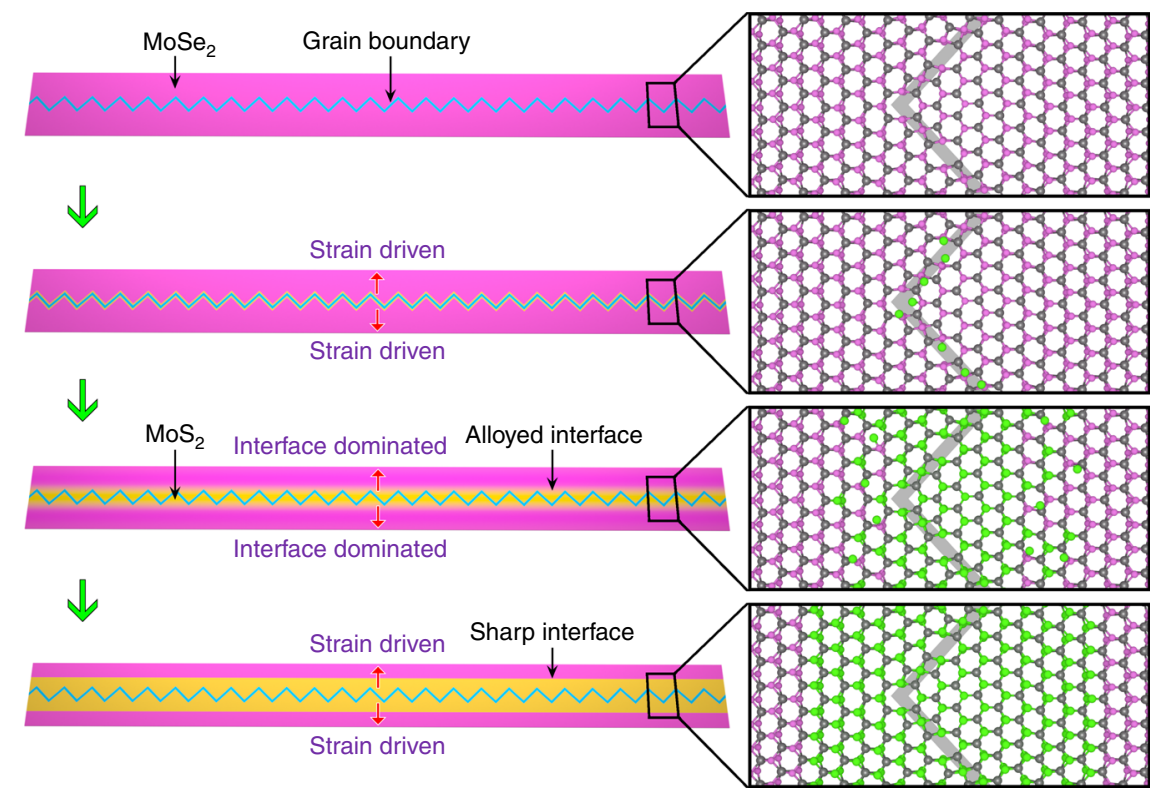

Fig. 1 Formation of ultra-long $\mathbf{M o S}_{\mathbf{2}}$ nano-channels. Schematic of growth steps of $\mathrm{MoS}_{2}$ channels: (I) MoSe $e_{2}$ monolayer with intrinsic $4 \mid 8 \mathrm{GBs}$ is produced; (II) sulfur atoms are adsorbed and then nucleation happens due to the strain at GBs; (III) Se atoms are continuously substituted by $\mathrm{S}$ atoms from GBs to nearby area to form alloyed regions, and afterward the growth is dominated by interface energy; and (IV) MoS 2 channels with sharp interface form, and eventually the growth is sustained by the strain again to widen the channels. 

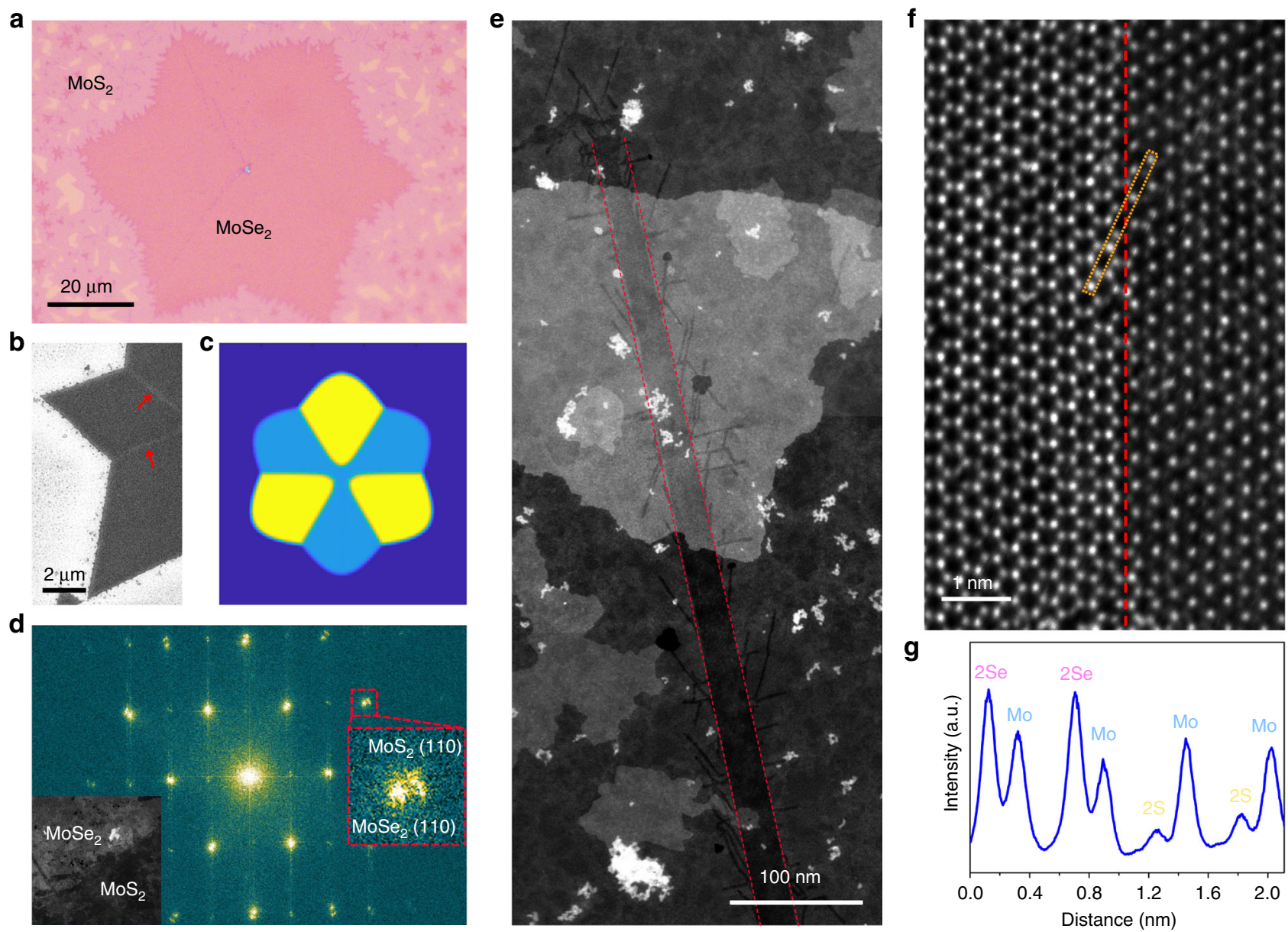

Fig. 2 Straight $\mathbf{M o S}_{\mathbf{2}}$ channels embedded in $\mathbf{M o S e}_{\mathbf{2}}$ matrix. a Optical image of the $\mathrm{MoS}_{2}-\mathrm{MoSe}_{2}$ lateral heterojunction, where monolayer MoS $\mathrm{S}_{2}$ surrounds the six-piont star $\mathrm{MoSe}_{2}$. b SEM image showing the location of nano-channels (noted by the arrows). c Phase-field simulation for the growth of six-point star $\mathrm{MoSe}_{2}$. d Fast Fourier transformation of the heterojunction region of the inserted ADF-STEM image. e Low-magnification ADF-STEM image showing a straight $\mathrm{MoS}_{2}$ channel of $>600 \mathrm{~nm}$ inside single-layer $\mathrm{MoSe}_{2}$. Some brighter patches are out-of-plane MoSe 2 introduced during the transfer process.

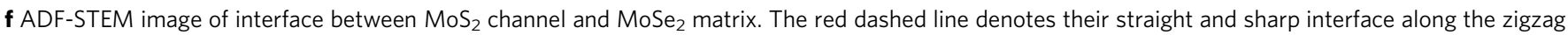
direction. $\mathbf{g}$ Line intensity profile of the rectangular region in $\mathbf{f}$

shows that the opposite sides of the straight channel have different lattice orientations. At each sidewall (left: Fig. 3f; right: Fig. 3h), S atoms in $\mathrm{MoS}_{2}$ channel seamlessly connect Mo atoms in $\mathrm{MoSe}_{2}$ matrix, forming a coherent lateral interface structure. Nevertheless, a $60^{\circ}$ (or mirror) lattice orientation difference between the opposite sides is observed, as demonstrated by the highlighted atoms in Fig. $3 \mathrm{f}, \mathrm{h}$. Careful examination of ADFSTEM images reveals that this orientation difference can be ascribed to the $60^{\circ} \mathrm{GB}$ in the middle region of the channel. The structure of the GB is labeled in Fig. $3 \mathrm{~g}$, which consists of successive fourfold and eightfold (4|8) rings. In our observation, the separated grains are mainly jointed in three ways (Supplementary Fig. 2): (I) S atoms from one grain bond Mo atoms from the other; (II) the grains share the same fourfold coordinative S atoms; (III) the grains meet at twofold coordinative $S$ atoms. Bonds type I are found in the paired fourfold and eightfold rings, while bonds type II exist between neighboring fourfold rings, consistent with theoretical predictions and other experimental findings in $\mathrm{TMDs}^{21,26}$. However, bonds type III that locate between neghbouring parallel eightfold rings is seldom reported. The ring configuration is directly related to the arrangement of each type of bonds, and according to the statistical results, the amount ratio between eightfold and fourfold rings is roughly 1:1.16 (Supplementary Fig. 3). Such a ratio that is higher than previous observation ${ }^{26}$ should be owing to some short chains of parallel eightfold rings (Supplementary Fig. 3b).

Then, we apply the geometric phase analysis (GPA) to draw the strain fields and rotation map (see Methods section and Supplementary Fig. 4) of the channel and surrounding matrix, as plotted in Fig. 3b-e. All the phase images are obtained with reference to the $\mathrm{MoSe}_{2}$ lattice. $\mathrm{MoS}_{2}$ channel presents compressive $\varepsilon_{x x}$ strain (normal strain along $x$-direction) compared with the surrounding $\mathrm{MoSe}_{2}$ matrix, despite that a tensile strained line is also found at the GB (Fig. 3b). For the majority area of $\mathrm{MoS}_{2}$ channel, the calculated compressive strain is in the range of $3.9 \pm$ $1.1 \%$, which corresponds to the $4.2 \% \mathrm{MoS}_{2}-\mathrm{MoSe}_{2}$ lattice mismatch, indicating a relaxed $\mathrm{MoS}_{2}$ lattice along the $x$-direction. In contrast, $\mathrm{MoS}_{2}$ channel and $\mathrm{MoSe}_{2}$ host show a uniform $\varepsilon_{y y}$ strain (normal strain along $y$-direction) phase except the GB region (Fig. 3c), suggesting $\mathrm{MoS}_{2}$ lattice is stretched to accommodate that of $\mathrm{MoSe}_{2}$ in the $y$-direction. The shear strain $\left(\varepsilon_{x y}\right)$ and lattice rotation are concentrated along the GB, as shown in Fig. $3 d$, e. From the rotation map, it can be clearly recognized that the GB possesses a zigzag extending trend, where the direction changing of GB leads to the altering of local orientations. Another obvious feature of the straight channel is that there are some branched $\mathrm{MoS}_{2}$ quantum wells at the heterojunction sidewalls (Figs. 2e and 3a). These 2-nm-wide 
a

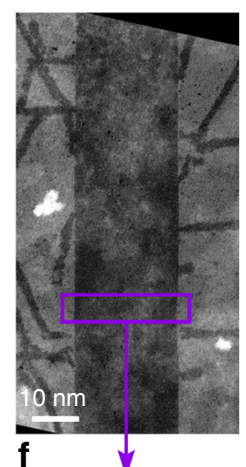

f b

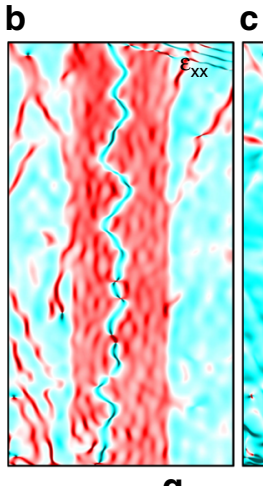

c

d

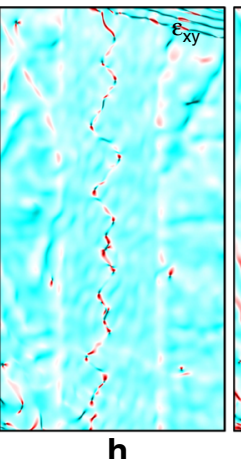

e
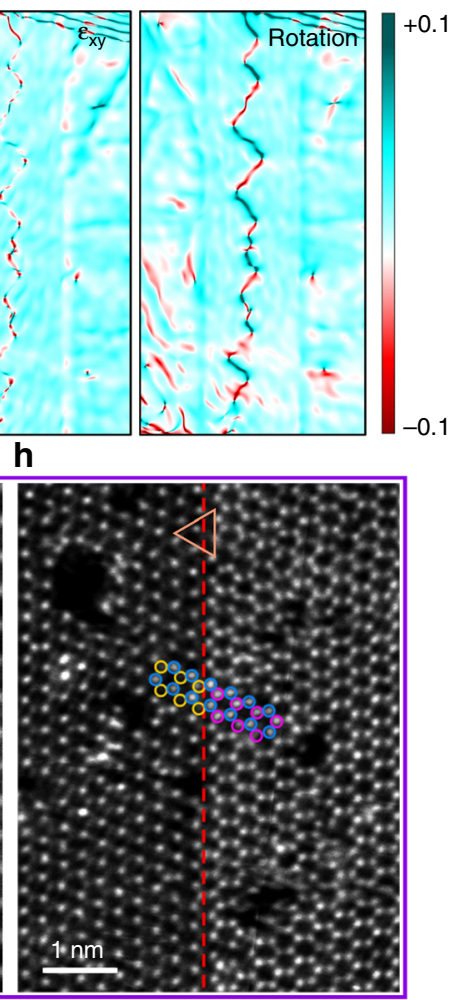

Fig. 3 Strain maps and structure analysis of straight nano-channels. a Low-magnification ADF-STEM image showing a part of straight MoS $\mathrm{S}_{2}$ nanochannel with the width of $\sim 20 \mathrm{~nm}$. b-e The corresponding strain (b-d) and rotation (e) maps of the channel area, showing the lattice strain and local orientations with respect to reference lattice $\left(\mathrm{MoSe}_{2}\right)$. A zigzag GB can be found at the middle of the straight channel. $\mathbf{f}-\mathbf{h}$ Atomically resolved ADF-STEM images of the rectangular region in $\mathbf{a}$. Red dashed lines denote the left (f) and right $(\mathbf{h})$ interface of the channel, respectively. Orange dashed line in $\mathbf{g}$ marks the $60^{\circ} \mathrm{GB}$ within the channel, and it consists of alternate $4 \mid 8$ rings as indicated by green tetragons and octagons. The lattice orientations at two sides of GB can be recognized from the triangles on the top and the highlighted atoms by circles (Mo, blue; Se, purple; S, yellow).

quantum wells grow along an armchair direction and always have $30^{\circ}$ or $90^{\circ}$ angle with the channel (Supplementary Fig. 5). The single dislocation (5/7 rings) -driven growth of such quantum wells has been investigated in recent literature ${ }^{25,27}$. It should be noticed that $\mathrm{MoS}_{2}$ nano-channels without branched quantum wells can also be obtained through the adjustment of experimental parameters (Supplementary Fig. 6), indicating the irrelevance of these two structures.

Besides the straight channels, winding ones are also observed. A winding channel usually has a non-uniform width ranging from 2 to $20 \mathrm{~nm}$ (Fig. 4a). Figure $4 \mathrm{~b}$ shows a superimposed image of the winding channel (enlarged area in Fig. 4a) and its rotation map, which complies with the extending trend of GB. By scanning the whole $\mathrm{GB}$, we find that the $\mathrm{GB}$ segments showing the $107 \pm 3^{\circ}$ angle tend to be a periodic zigzag shape with no change of the overall GB direction, while those with $79^{\circ}$ and $141^{\circ}$ angles serve as a kink that turn the overall direction of the GB. To further illustrate this point, we construct a model for the GB segment with a $107^{\circ}$ angle in Supplementary Fig. 7, where mixed $4 \mid 8$ and $8|4| 4 \mid 8$ dislocations are neatly assembled along the GB. Such a GB is actually a consequence of seamless coalescence of two domains with mirror symmetry and has notably higher stability than those with other atomic organizations. For example, a comparison of such a folded GB with one composed fully of $8|4|$ $4 \mid 8$ dislocations $\left(97^{\circ}\right)$ shows lower formation energy of 20-77 $\mathrm{meV} \AA^{-1}$ in the whole range of the chemical potential of sulfur. The preferred atomic organization around the GB segments folded with a $107^{\circ}$ angle leads to their prevalence in our experimental observations. Aa a result, the winding channel has a step-like interface with surrounding $\mathrm{MoSe}_{2}$ (Fig. 4c), probably because its extending direction is not along the zigzag direction of lattice for most cases, which may also lead to the width variation of winding channel. Except for this difference, the atomic structure of the winding channel (Fig. 4c) resembles that of the straight one (Fig. $3 \mathrm{f}-\mathrm{h}$ ). In addition, winding and straight channels can connect with each other (Fig. 4d, Supplementary Fig. 8), suggest the similar nature of these two kinds of channels.

Strain-driven growth mechanism based on GB. To gain an insight into the growing mechanism, the atomic substitution process near the GBs is carefully studied by first-principles calculations. For simplicity, we consider an individual $S$ atom for substitution reaction. In a real situation, the S-containing species may be more complicated, but the reaction is essentially the replacement of Se atoms with S atoms. Generally, the substitution of Se with $S$ includes three steps, i.e., the chemisorption of sulfur on the $\mathrm{MoSe}_{2}$ surface, an intermediate state with $\mathrm{S}$ and Se atoms being about to swap with each other, and the detachment of Se atoms, as illustrated by the insets in Fig. 5a. Overall, the substitution reaction is thermodynamically favorable given the higher energy of step-one configuration than the step-three one. Among the three steps, the second step is found to have the highest energy, indicating that it is the rate-limiting step throughout the whole reaction. Our proof calculations based on the energy barrier are in the same trend as that based on $\Delta E$, that is a higher $\Delta E$ correspond to a higher energy barrier presented in Supplementary Fig. 9. Apparently, a reaction site that can 

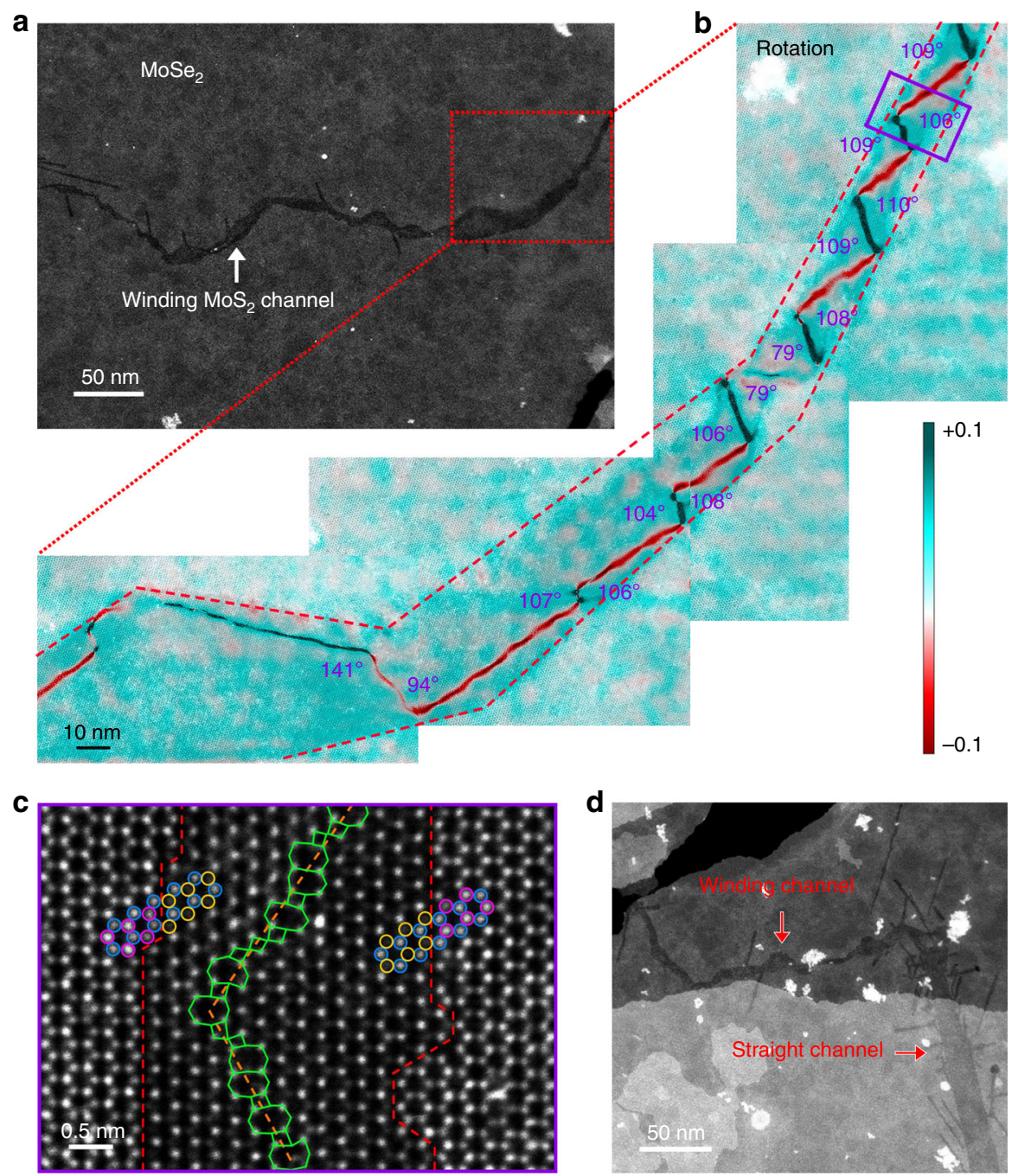

Fig. 4 Rotation map and structure analysis of winding $\mathbf{M o S}_{\mathbf{2}}$ channels. a Low-magnification ADF-STEM image of a winding MoS 2 channels. $\mathbf{b}$ The superimposed image of the channel and its corresponding rotation map. $\mathbf{c}$ High-resolution ADF-STEM image showing the atomic structure of the rectangular area in $\mathbf{b}$. The heterojunction interface is highlighted by the red dashed lines. $60^{\circ} \mathrm{GB}$ (orange dashed line) is formed by $4 \mid 8$ rings (green tetragons and octagons). Blue, purple, and yellow circles represent Mo, Se, and S atoms. d ADF-STEM image of a winding channel connecting with a straight channel.

utmostly stabilize the intermediate state could ultimately favor the reaction relative to other sites.

With this provision, we can use the relative energy of the intermediate state, $\Delta E$ (with respect to the most stable reaction site), as a reference to examine the nucleation selectivity of the $\mathrm{MoS}_{2}$ channels and their dynamical evolution via the sulfur substitution. For this purpose, we first consider a GB model composed of $8|4| 4 \mid 8$ dislocations, the most frequently observed GB type in our experiments. A careful scan of all possible reaction sites near the GB shows that the Se atom shared by two squares will be the first to be substituted by $S$ atom, marked by 1 in Fig. 5b. In particular, $\Delta E$, exhibits a sharp minimum of up to 1.8 $\mathrm{eV}$ deep as the reaction site moves toward the GB from the perfect lattice region (Fig. $5 \mathrm{c}$ ). The strong propensity of the substitution reaction at the GB is attributed to the high in-plane tensile $\operatorname{strain}^{28,29}$ therein (see the strain map in Fig. 3b and Supplementary Fig. 10), which greatly lowers the energy of the intermediate state due to the alleviated steric effect, while leaving the step-one configuration less influenced. Then, the $\mathrm{S}$ atom in $\mathrm{MoSe}_{2}$ will serve as the nucleation center for the continuous growth of the $\mathrm{MoS}_{2}$. Indeed, the subsequent substitutions are energetically preferred at the Se site adjacent to the substituted $\mathrm{S}$ atom (marked by 2 and 3 in Fig. 5b). With a continuous supply of $\mathrm{S}$ atoms, the substitution of Se will not only strictly follow the GB but also extend toward the perfect lattice region. This trend is well illustrated by a contour plot of the sequence of $S$ substitution in the $\mathrm{MoSe}_{2}$ layer as shown in Fig. 5d, based on extensive calculations. This plot is essentially a translated version of the strain map around the GB. Such a strain-driven reaction mechanism well explains the $\mathrm{MoS}_{2}$ channel growth in our experiments.

The growing mechanism discussed above is supported by STEM investigations at different growth steps of the GBs-based channels. Figure 6a, b displays a pristine $\mathrm{MoSe}_{2}$ monolayer before $S$ substitution (Methods section), where the same structured $60^{\circ}$ GB composed of $4 \mid 8$ rings is recognized. In addition, these GBs are located between two neighboring corners of six-point star $\mathrm{MoSe}_{2}$, identical to the locations of channels in Fig. 2b. Both verify that the $60^{\circ} \mathrm{GBs}$ are intrinsic defects in pristine $\mathrm{MoSe}_{2}$ rather than introduced structures during the growth of $\mathrm{MoS}_{2}$ 

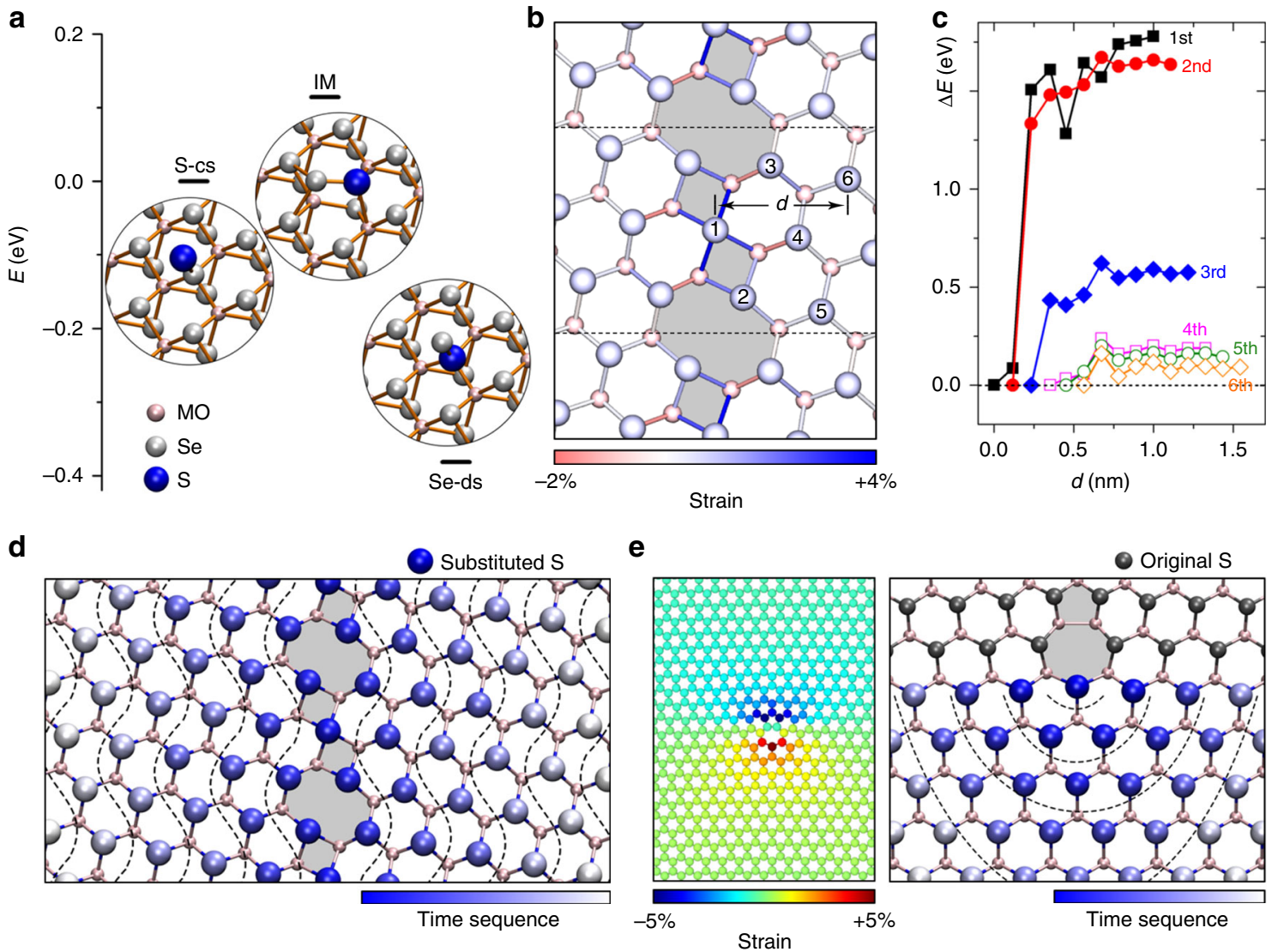

Fig. 5 Growth mechanism of the $\mathbf{M o S}_{\mathbf{2}}$ channels. a Reaction steps for the selenium substitution with sulfur and their relative energies. $\mathbf{b}$ Atomic structure of an $8|4| 4 \mid 8 \mathrm{~GB}$ along with map of bond strain induced by the GB. The numbers 1-6 denote the sequence of sulfur substitution. $d$ denotes the distance of reaction sites with respect to the GB line. c Calculated relative energies $\Delta E$ for the intermediate state in a as a function of $d$ for the 1-6 sulfur atoms along the optimal substitution pathway. d Contour plot of substitution sequence (from blue to light gray) around the $8|4| 4 \mid 8 \mathrm{~GB}$. e In-plane strain distribution near a misfit Mo-rich 5/7 dislocation and the corresponding contour plot for the sequence of substitution reaction.

channels. After supplying S for a short time at $\sim 700^{\circ} \mathrm{C}$, some Se atoms near the GB are substituted by $S$ atoms to form a $\mathrm{MoSe}_{2}-\mathrm{MoS}_{2}$ hybrid channel (Fig. 6c). Although $\mathrm{MoSe}_{2}$ has completely transformed into $\mathrm{MoS}_{2}$ near the core area of the GB, there are some alloyed structures near the interfaces. The intensity profile (insert in Fig. 6d and Supplementary Fig. 11) confirms that one Mo atom randomly coordinates with $\mathrm{S}_{2}, \mathrm{Se}_{2}$, or $\mathrm{S}+\mathrm{Se}$ atoms in the alloyed regions, as labeled by the arrows in Fig. 6d. Yet, no alloyed structure is observed in the region away from the interfaces, implying that GBs are the nucleation sites for the channel growth. Moreover, some chemisorptive $\mathrm{S}$ atoms can be identified at the $\mathrm{MoSe}_{2}-\mathrm{MoS}_{2}$ interface (Supplementary Fig. 12). These observations agree with our theoretical scenario that the S substitution starts at the GBs and then spreads toward the bulk regions, guided by the long-range strain fields pertaining to the GBs.

The strain-driven reaction mechanism can be applied to other sources of lattice strain, such as dislocations and point defects. Different lattice constants of $\mathrm{MoS}_{2}$ and $\mathrm{MoSe}_{2}$ result in a misfit Mo-rich 5/7 dislocation at their interface, which can induce an even higher long-range strain field than that by GBs, as evidenced by the strain map in the left panel of Fig. 5e. The calculated substitution sequence according to energy criteria reveals a strong preference of reaction at the dislocations and a substitution pathway closely following the strain map (Supplementary Fig. 13). As a result, the growth of $\mathrm{MoS}_{2}$ channels at the interface branch off from the dislocations, forming a unique sub-channel spreading into the $\mathrm{MoSe}_{2}$ areas, as observed experimentally in
Fig. 2e. Besides the dislocations, we also purposefully create Mo and Se vacancies in the $\mathrm{MoSe}_{2}$, which induce strain only in the immediate vicinity of the defects. As expected, the growth of $\mathrm{MoS}_{2}$ does occur near the defects but spreads only by several atoms away from the vacancies (Supplementary Fig. 14). We envision that the bias of chemical reaction can also be amenable to applied elastic strain, which potentially enables larger flexibility for fabricating $\mathrm{MoS}_{2}$ superstructures with a wide variety of patterns.

Furthermore, it is worth mentioning that the growth of $\mathrm{MoS}_{2}$ channels ends up with a sharp heterointerface after long-time reaction. This can be understood by the minimized interface energy. After the growth of channels advances far from the GB, the effect of GB-induced strain is diminished (Supplementary Fig. 15), and $\mathrm{MoSe}_{2}-\mathrm{MoS}_{2}$ interfaces gradually dominate the extension of the channels. As such, the growth will proceed in a manner that minimizes the interface energy, thereby resulting in a sharp, straight interface. Firstly, our DFT calculations prove that a zigzag-shaped $\mathrm{MoSe}_{2}-\mathrm{MoS}_{2}$ heterointerface will be gradually smoothened upon the substitution reaction and finally evolve into a straight line, as shown in Fig. 6e. Then, the strain always plays a major role in sustaining the growth of the $\mathrm{MoS}_{2}$ channel. At the sharp interface between $\mathrm{MoS}_{2}$ and $\mathrm{MoSe}_{2}$ in Supplementary Fig. 16, scanning all possible reaction sites shows that the Se atom closest to the interface will be the first to be substituted by $S$ atom, followed by the Se atoms on the same row, with $\Delta E$ exhibiting a sharp minimum of $0.05-0.15 \mathrm{eV}$ deep. The overall growth process of $\mathrm{MoS}_{2}$ channels are schematically illustrated in Fig. 1, vividly 

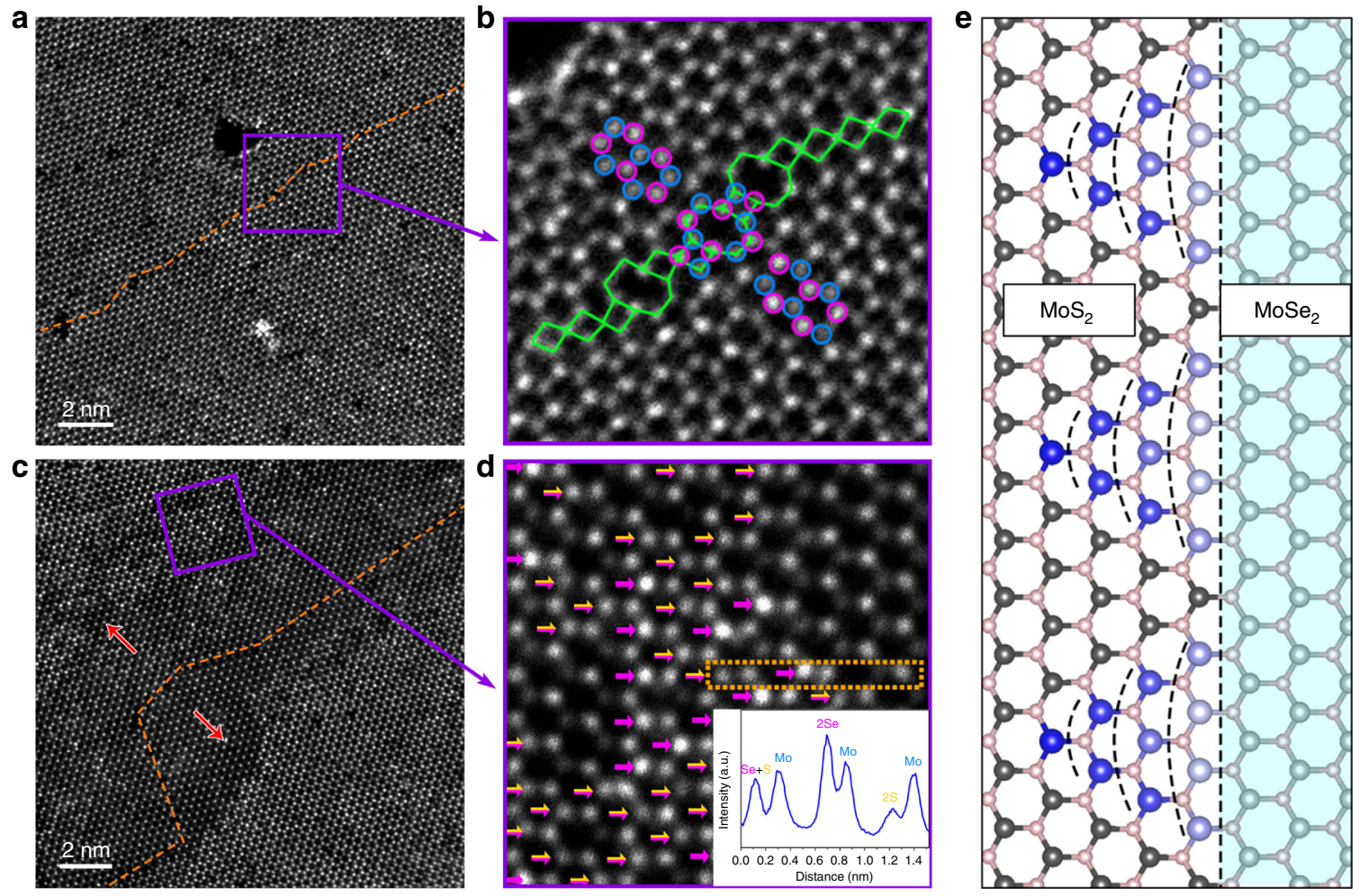

Fig. 6 Growth process of $\mathbf{M o S}_{\mathbf{2}}$ channels. a, b ADF-STEM images of intrinsic $60^{\circ} \mathrm{GB}$ (orange dashed line) within pristine monolayer MoSe ${ }_{2}$. Atomic arrangement of this GB (blue and purple circles: Mo and Se atoms; and green tetragons and octagons: 4 and 8 rings) resembles that in $\mathrm{MoS}_{2}$ nanochannels. c, d ADF-STEM images showing part of $\mathrm{MoSe}_{2}-\mathrm{MoS}_{2}$ hybrid winding channel. Some Se atoms has been replaced by $S$ atoms, as indicated in d (purple arrows mark $2 \mathrm{Se}$ atoms and yellow-purple arrows mark S + Se atoms). Line intensity profile of $2 \mathrm{Se}, 2 \mathrm{~S}, \mathrm{~S}+\mathrm{Se}$, and Mo atoms is provided in the insert of $\mathbf{d}$. The red arrows in c represent the sulfidation direction. e A contour plot for the sequence (from blue to light gray) of substitution reaction along a zigzag $\mathrm{MoSe}_{2}-\mathrm{MoS}_{2}$ interface.

displaying a transition from the GB-driven growth mechanism to an interface-dominated mechanism.

Catalytic performance of nano-channels for HER. It has been reported that GBs in TMDs possess better catalytic performance in hydrogen evolution reaction (HER) than intact lattice ${ }^{30}$. In addition, recent works also prove the connection between the catalytic activity and the strain of crystal lattice for kinds of $\mathrm{TMDs}^{31,32}$. In the consideration of these facts, it is reasonable to speculate that the combination of strain and GBs should be an effective strategy for further improvement of the activity in hydrogen production. To our delight, the $\mathrm{MoS}_{2}$ nano-channels are appropriate structures that applying a $y$-direction $3.9 \%$ latticemismatch strain along GBs (Fig. 3c). Moreover, it should be noticed that this method is a spontaneous process, only if any GB exists in $\mathrm{MoSe}_{2}, \mathrm{MoS}_{2}$ nano-channel can be produced accurately along the $\mathrm{GB}$ to introduce the lattice-mismatch strain, which cannot be accomplished by other external strain engineering strategies, such as stretching ${ }^{33}$ and bending ${ }^{34}$. In order to investigate the activity of the spontaneous strained $\mathrm{MoS}_{2}$ nanochannels, we develop a micro-electrochemical device to exactly examine the hydrogen production performance (Supplementary Fig. 17), as shown in Fig. 7a. For comparison, the same devices are also fabricated on common single GB and basal plane in pure $\mathrm{MoS}_{2}$ (Supplementary Fig. 18). Figure 7b, c presents the polarization curves and the corresponding Tafel slopes in $0.5 \mathrm{M} \mathrm{H}_{2} \mathrm{SO}_{4}$ solution, respectively, for three different devices ( $\mathrm{Pt}$ is also included as a reference). It can be seen that although the common GB in pure $\mathrm{MoS}_{2}$ has achieved a better catalytic activity than the basal plane, the spontaneous strained nano-channel even exhibits a more improved performance. The statistical data (Fig. $7 \mathrm{~d}$ ) based on tens of devices strengthen the reliability of our results, confirming that the spontaneous strain in $\mathrm{MoS}_{2}$ nano-channels can further improve the catalytic activity of GBs.

\section{Discussion}

In conclusion, we have demonstrated the growth of ultra-long nano-channels based on GBs, and the length of the nanochannels is comparable with the matrix. We proposed a straindriven growing mechanism to elucidates the role of defects (both point and line defects) in the formation of the nano-channels. The nano-channels have also been proved to introduce spontaneous strain at GBs for efficient improvement of catalytic performance in hydrogen production, and also for bandgap altering (Supplementary Fig. 19). Our work has suggested a different strategy to realize narrow heterostructures with great potential for bandgap engineering, quantum effect, and electrocatalysis such as the formation of $1 \mathrm{D}$ charge density waves ${ }^{35}$, gate-tunable memristive phenomena ${ }^{36}$, and strong photoluminescence quenching/ enhancement ${ }^{26}$.

\section{Methods}

Synthesis procedure. The $\mathrm{MoS}_{2}-\mathrm{MoSe}_{2}$ lateral heterostructures were synthesized by a two-step chemical vapor depostion (CVD) method using $\mathrm{MoO}_{3}$, Se, and $\mathrm{S}$ powders as the Mo, Se, and S source, respectively. The first step: the growth of $\mathrm{MoSe}_{2}$ flakes. Specifically, a clean $\mathrm{SiO}_{2} / \mathrm{Si}$ substrate was placed face-down onto a quartz boat containing $15 \mathrm{mg} \mathrm{MoO}_{3}$ powders. The boat was loaded into the middle of a quartz tube with an inner diameter of 1 inch. Another quartz boat containing $0.5 \mathrm{~g}$ Se powder was placed upstream. The growth was performed at atmospheric pressure with a mixture of $5 \mathrm{sccm} \mathrm{H}_{2}$ and $50 \mathrm{sccm} \mathrm{Ar}$ as the carrier gas. The 
a

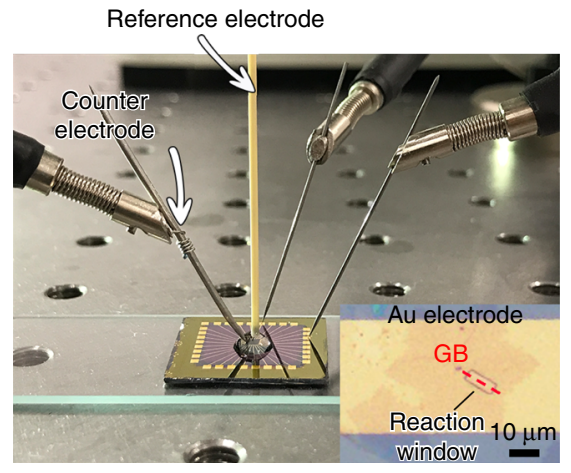

b
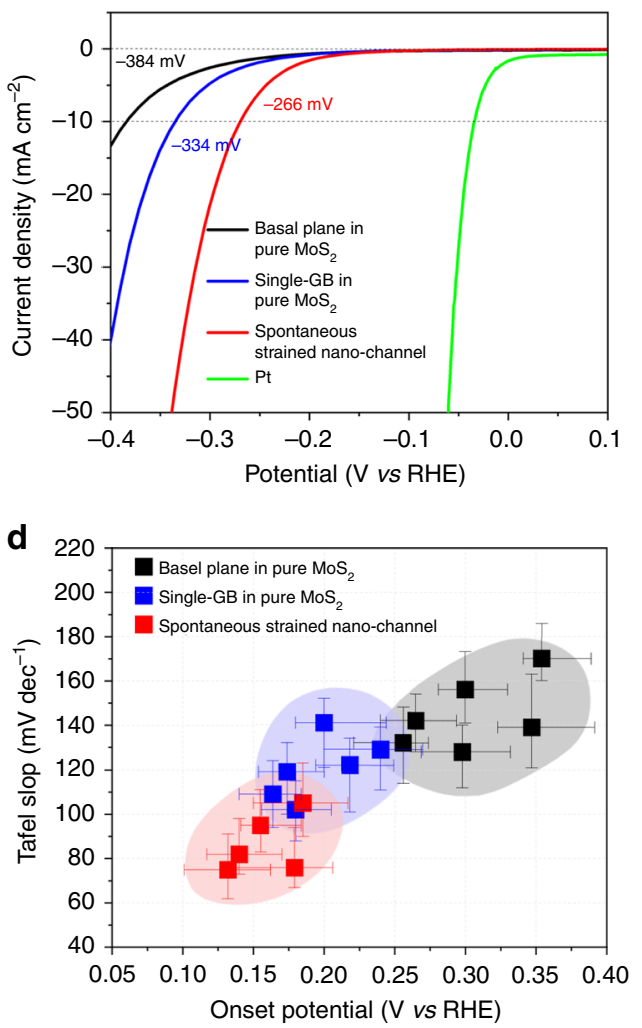

Onset potential ( $\mathrm{V}$ vs RHE)

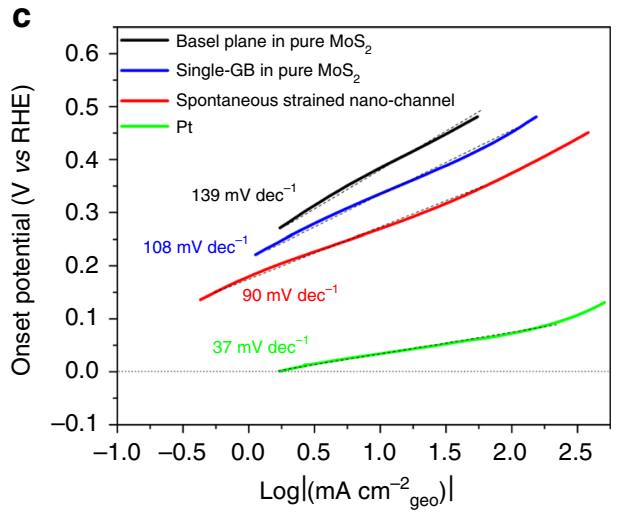

Fig. 7 HER activity of $\mathbf{M o S}_{\mathbf{2}}$ single GB in nano-channel. a Photograph of micro-electrochemical cell. Inset: device with a micro-size reaction window at the spontaneous strained nano-channel. The hydrogen evolution reaction (HER) only occurs within the reaction window, and the rest of the areas are passivated by electrochemically inert PMMA film. b-c Polarization curves of the current density (b) and the corresponding Tafel plots (c) of the devices for spontaneous strained $\mathrm{MoS}_{2}$ nano-channel, single GB, and basal plane in pure $\mathrm{MoS}_{2}$, respectively. d Statistical HER results obtained from dozens of microelectrochemical devices. The error bars define the data statistical range of multiple measurements for each micro-electrochemical cell.

temperature ramped up to $720^{\circ} \mathrm{C}$ at a rate of $30^{\circ} \mathrm{C} \mathrm{min}^{-1}$, and the duration time at $720^{\circ} \mathrm{C}$ was set to $10 \mathrm{~min}$. After that, the furnace was cooled down to room temperature naturally. The second step: the growth of heterostructures. Specifically, the cooled substrate was immediately transferred to another quartz tube for $\mathrm{MoS}_{2}$ monolayer growth. During this step, $15 \mathrm{mg} \mathrm{MoO}_{3}$ and $0.5 \mathrm{~g} \mathrm{~S}$ powder were used as the precursors and the gas flow kept the same as in the $\mathrm{MoS}_{2}$ case. The growth was carried out at $700^{\circ} \mathrm{C}$ for $5 \mathrm{~min}$.

STEM sample preparation. The STEM samples were transferred using a poly (methyl methacrylate) (PMMA) assisted method. A thin layer of $1 \mu \mathrm{m}$ PMMA was spin coated onto the $\mathrm{SiO}_{2} / \mathrm{Si}$ substrate and then baked at $120^{\circ} \mathrm{C}$ for $3 \mathrm{~min}$. The substrate was immersed in $20 \% \mathrm{KOH}$ solution to etch the $\mathrm{SiO}_{2}$ layer, resulting in a floating PMMA/MoS $2-\mathrm{MoSe}_{2}$ film. After washing with deionized water, the film was fished by a Au TEM grid (Quantifoil, $50 \mathrm{~nm}$ Au foil of 300 mesh). Finally, PMMA coating layer was dissolved by soaking the grid in $60^{\circ} \mathrm{C}$ acetone for $3 \mathrm{~h}$. Before STEM characterization, the grid was annealed under Ar atmosphere at $200^{\circ} \mathrm{C}$ for $3 \mathrm{~h}$ to avoid carbon hydrocarbon contamination.

STEM characterization. STEM characterization was carried out on a JEOL ARM200F (S)TEM equipped with CEOS CESCOR aberration corrector, operated at an accelerating voltage of $80 \mathrm{kV}$. The convergence semi-angle and acquisition semiangle were $28-33$ and $68-280$ mrad for the ADF imaging. The dwell time per pixel was set to $12-20 \mu$ s. The atomic resolution ADF images were deconvolution filtered using Richard-Lucy method to enhance the contrast.

Geometric phase analysis. GPA is a standard method to quantitatively extract displacement fields and strain maps from high-resolution TEM images. This method is based on the Fourier transformation and inverse Fourier transformation because the lattice information of real space is described by the corresponding peaks in the reciprocal space. Here, we applied GPA to map the strain fields of $\mathrm{MoS}_{2}$ nano-channels embedded in $\mathrm{MoSe}_{2}$ matrix. Firstly, two strong nonparallel Bragg reflections $\mathbf{g}_{1}$ and $\mathbf{g}_{2}$ in FFT images are selected and covered by Gaussian masks (Supplementary Fig. 4b). The followed calculation was performed to generate the phase images (Supplementary Fig. 4c, d) and scale of reciprocal lattice (Supplementary Fig. 4e, f) corresponding to reflections $\mathbf{g}_{\mathbf{1}}$ and $\mathbf{g}_{2}$, as well as the strain and rotation maps (Supplementary Fig. $4 \mathrm{~g}-\mathrm{j}$ ).

Theoretical calculations. All the theoretical calculations were performed with the Vienna Ab-initio Simulation Package ${ }^{37}$. The projector-augmented wave method for the core region and the generalized gradient approximation with the Perdew, Burke, and Ernzerhof functional were employed. Kinetic energy cutoff of $300 \mathrm{eV}$ was adopted in the plane-wave expansion. The model systems are constructed in nanoribbon configurations, in which the distance between GBs and edges is $\sim 7 \mathrm{~nm}$, large enough to achieve the convergence of the energies. All structures are fully relaxed until the force on each atom is $<0.01 \mathrm{eV} \AA^{-1}$. The Brillouin zone integration was sampled by five special $k$-points along the periodic orientation for the $8|4| 4 \mid 8 \mathrm{GBs}$ and gamma-only points for the $5 \mid 7$ dislocations.

The phase-field setups are almost identical to that in ref. ${ }^{38}$. We digest the adaptions we adopted for the current simulation. The model ${ }^{39,40}$ starts from a free energy functional,

$$
G=\sum_{\alpha, \beta=1,2, \alpha<\beta} \frac{4 \sigma_{\alpha \beta}}{\pi^{2}}\left(-\eta \nabla \phi_{\alpha} \times \nabla \phi_{\beta}+\frac{\pi^{2}}{\eta} \phi_{\alpha} \phi_{\beta}\right)+\lambda \xi h\left(\phi_{N}\right)
$$

Variables description can be found in the publication ${ }^{38}$. In the current simulation, we set $N=3$ thus two grain orientations are considered. The simulation early stage is virtually the growth of a David $\operatorname{star}^{38}$ on a $256 \times 256$ canvas. Each nucleus starts from a circle with a radius of $3 l$. Distances between central nucleus and satellite nuclei are $10 \mathrm{l}$. Once the length of David star reaches $85 \%$ of edge length, the flux is set to zero so the growth ceases and etching starts. The simulation stops when the length of David star drops to $30 \%$ of edge length. The parameters are listed in Supplementary Table 1.

Fabrication of micro-electrochemical device and electrocatalytic measurement. First, a prepatterned set of $32 \mathrm{Au}$ contact pads was fabricated on a $16 \mathrm{~mm} \times$ $16 \mathrm{~mm} \mathrm{SiO} 2(285 \mathrm{~nm}) / \mathrm{Si}$ chip using conventional photolithography (Supplementary Fig. 17a). Second, $\mathrm{MoS}_{2} / \mathrm{MoS}_{2}-\mathrm{MoSe}_{2}$ film from the CVD growth method was 
transferred onto this chip (Supplementary Fig. 17b), and a further annealing process at $200^{\circ} \mathrm{C}$ under high-vacuum conditions $\left(1 \times 10^{-5}\right.$ torr $)$ was employed to optimize their interfaces to facilitate the electron vertical injection from Au to the sample during reaction (Supplementary Fig. 17c). Third, a 1- $\mu$ m-thick PMMA film was coated on the device chip, and an e-beam lithography process was followed to open a reaction window on this film to expose the region of interest on the nanosheet for reaction (Supplementary Fig. 17d). The location of single GB can be clearly identified by Raman mapping or dark-field optical microscope.

A micro-electrochemical device with four electrodes was adopted in the electrocatalytic experiment (Fig. 7a). In all measurements, only the exposed region of the sample contributes to HER performance. The measurements were conducted in a $0.5 \mathrm{M} \mathrm{H}_{2} \mathrm{SO}_{4}$ electrolyte solution. The scan rate was set to be $5 \mathrm{mv}$ per step. The electrocatalytic current $\left(I_{\mathrm{c}}\right)$ and conductance current $\left(I_{\mathrm{ds}}\right)$ are simultaneously detected.

\section{Data availability}

The data that support the plots within this paper and other findings of this study are available from the corresponding authors upon reasonable request

Received: 8 March 2019; Accepted: 9 January 2020;

Published online: 07 February 2020

\section{References}

1. Liu, Y. et al. Van der Waals heterostructures and devices. Nat. Rev. Mater. 1, 16042 (2016)

2. Chiu, M.-H. et al. Determination of band alignment in the single-layer MoS2/ WSe2 heterojunction. Nat. Commun. 6, 7666 (2015).

3. Duan, X., Wang, C., Pan, A., Yu, R. \& Duan, X. Two-dimensional transition metal dichalcogenides as atomically thin semiconductors: opportunities and challenges. Chem. Soc. Rev. 44, 8859-8876 (2015).

4. Sun, Z., Martinez, A. \& Wang, F. Optical modulators with 2D layered materials. Nat. Photonics 10, 227-238 (2016).

5. Zhang, X.-Q., Lin, C.-H., Tseng, Y.-W., Huang, K.-H. \& Lee, Y.-H. Synthesis of lateral heterostructures of semiconducting atomic layers. Nano Lett. 15, 410-415 (2014).

6. Chen, K. et al. Lateral built-in potential of monolayer MoS2-WS2 in-plane heterostructures by a shortcut growth strategy. Adv. Mater. 27, 6431-6437 (2015).

7. Li, M.-Y. et al. Epitaxial growth of a monolayer WSe2-MoS2 lateral pn junction with an atomically sharp interface. Science 349, 524-528 (2015).

8. Zhang, J., Xie, W., Zhao, J. \& Zhang, S. Band alignment of two-dimensional lateral heterostructures. 2D Mater. 4, 015038 (2016).

9. Kang, J., Tongay, S., Zhou, J., Li, J. \& Wu, J. Band offsets and heterostructures of two-dimensional semiconductors. Appl. Phys. Lett. 102, 012111 (2013).

10. Duan, $X$. et al. Lateral epitaxial growth of two-dimensional layered semiconductor heterojunctions. Nat. Nanotechnol. 9, 1024-1030 (2014).

11. Gong, Y. et al. Vertical and in-plane heterostructures from WS2/MoS2 monolayers. Nat. Mater. 13, 1135-1142 (2014).

12. Bogaert, K. et al. Diffusion-mediated synthesis of MoS2/WS2 lateral heterostructures. Nano Lett. 16, 5129-5134 (2016).

13. Gong, Y. et al. Two-step growth of two-dimensional WSe2/MoSe2 heterostructures. Nano Lett. 15, 6135-6141 (2015).

14. Ullah, F. et al. Growth and simultaneous valleys manipulation of twodimensional MoSe2-WSe2 lateral heterostructure. ACS Nano 11, 8822-8829 (2017).

15. Zhang, Z. et al. Robust epitaxial growth of two-dimensional heterostructures, multiheterostructures, and superlattices. Science 357, 788-792 (2017).

16. Sahoo, P. K., Memaran, S., Xin, Y., Balicas, L. \& Gutiérrez, H. R. One-pot growth of two-dimensional lateral heterostructures via sequential edgeepitaxy. Nature 553, 63-67 (2018).

17. Zhou, J. et al. A library of atomically thin metal chalcogenides. Nature $\mathbf{5 5 6}$ 355-359 (2018).

18. Gao, Y. et al. Ultrafast growth of high-quality monolayer WSe2 on Au. Adv. Mater. 29, 1700990 (2017).

19. Hafeez, M., Gan, L., Li, H., Ma, Y. \& Zhai, T. Large-area bilayer ReS2 film/ multilayer ReS2 flakes synthesized by chemical vapor deposition for high performance photodetectors. Adv. Funct. Mater. 26, 4551-4560 (2016).

20. Zhou, W. et al. Intrinsic structural defects in monolayer molybdenum disulfide. Nano Lett. 13, 2615-2622 (2013).

21. Zou, X., Liu, Y. \& Yakobson, B. I. Predicting dislocations and grain boundaries in two-dimensional metal-disulfides from the first principles. Nano Lett. 13, 253-258 (2013).

22. Huang, Y. L. et al. Bandgap tunability at single-layer molybdenum disulphide grain boundaries. Nat. Commun. 6, 6298 (2015).
23. Azizi, A. et al. Dislocation motion and grain boundary migration in twodimensional tungsten disulphide. Nat. Commun. 5, 4867 (2014).

24. Zhang, Z., Zou, X., Crespi, V. H. \& Yakobson, B. I. Intrinsic magnetism of grain boundaries in two-dimensional metal dichalcogenides. ACS Nano 7 , 10475-10481 (2013).

25. Han, Y. et al. Sub-nanometre channels embedded in two-dimensional materials. Nat. Mater. 17, 129-133 (2018).

26. Van Der Zande, A. M. et al. Grains and grain boundaries in highly crystalline monolayer molybdenum disulphide. Nat. Mater. 12, 554-561 (2013).

27. Zhou, W. et al. Dislocation-driven growth of two-dimensional lateral quantum-well superlattices. Sci. Adv. 4, eaap9096 (2018).

28. Wei, Y. et al. The nature of strength enhancement and weakening by pentagon-heptagon defects in graphene. Nat. Mater. 11, 759-763 (2012)

29. Zhang, T., Li, X. \& Gao, H. Defects controlled wrinkling and topological design in graphene. J. Mech. Phys. Solids 67, 2-13 (2014).

30. Zhu, J. et al. Boundary activated hydrogen evolution reaction on monolayer MoS2. Nat. Commun. 10, 1348 (2019).

31. Li, H. et al. Activating and optimizing MoS2 basal planes for hydrogen evolution through the formation of strained sulphur vacancies. Nat. Mater. 15 48-53 (2016).

32. Zhao, S. et al. Group VB transition metal dichalcogenides for oxygen reduction reaction and strain-enhanced activity governed by p-orbital electrons of chalcogen. Nano Res. 12, 925-930 (2019).

33. Castellanos-Gomez, A. et al. Local strain engineering in atomically thin MoS2. Nano Lett. 13, 5361-5366 (2013).

34. Zhang, Q. et al. Strain relaxation of monolayer WS2 on plastic substrate. Adv Funct. Mater. 26, 8707-8714 (2016).

35. Barja, S. et al. Charge density wave order in 1D mirror twin boundaries of single-layer MoSe2. Nat. Phys. 12, 751-756 (2016).

36. Sangwan, V. K. et al. Gate-tunable memristive phenomena mediated by grain boundaries in single-layer MoS2. Nat. Nanotechnol. 10, 403-406 (2015).

37. Kresse, G. \& Furthmüller, J. Efficient iterative schemes for ab initio totalenergy calculations using a plane-wave basis set. Phys. Rev. B 54, 11169 (1996)

38. Artyukhov, V. I., Hu, Z., Zhang, Z. \& Yakobson, B. I. Topochemistry of bowtie-and star-shaped metal dichalcogenide nanoisland formation. Nano Lett. 16, 3696-3702 (2016).

39. Steinbach, I. \& Pezzolla, F. A generalized field method for multiphase transformations using interface fields. Phys. D 134, 385-393 (1999).

40. Karma, A. \& Rappel, W.-J. Quantitative phase-field modeling of dendritic growth in two and three dimensions. Phys. Rev. E 57, 4323-4349 (1998).

\section{Acknowledgements}

This work is supported by the Singapore National Research Foundation under NRF award number NRF-RF2013-08, MOE Tier 2 MOE2016-T2-2-153, MOE2015-T2-2-007, MOE2015-T2-2-043, and MOE2018-T3-1-002. The part of work in NUAA is supported by NSF of China $(51535005,51472117$, and 11772153), the Research Fund of State Key Laboratory of Mechanics and Control of Mechanical Structures (MCMS-I-0418K01, MCMS-0417G02, and MCMS-0417G01). The part of work in UCAS is supported by NSF of China (51622211 and 51872285).

\section{Author contributions}

C.Z. and Z.L. conceived the idea. C.Z. performed the electron microscopy experiments, electron microscopy data analysis, and wrote the paper. M.Y., Z.Z., Z.H., and W.G. performed the theoretical calculations and participated in the paper writing. J.Z. synthesized the $\mathrm{MoSe}_{2}-\mathrm{MS}_{2}$ heterostructure samples. Y.H., Y.D., and S.G. carried out the electrochemical experiment. W.Z., C.Z., M.X., and J.S. performed the electron energy loss spectroscopy (EELS) experiment. Q.Z., L.W., and L.S. participate in the data analysis. All authors discussed the results and commented on the manuscript.

\section{Competing interests}

The authors declare no competing interests.

\section{Additional information}

Supplementary information is available for this paper at https://doi.org/10.1038/s41467 020-14521-8.

Correspondence and requests for materials should be addressed to Z.Z., W.G. or Z.L.

Peer review information: Nature Communications thanks Meizhuang Liu and the other, anonymous, reviewer(s) for their contribution to the peer review of this work. Peer reviewer reports are available

Reprints and permission information is available at http://www.nature.com/reprints

Publisher's note Springer Nature remains neutral with regard to jurisdictional claims in published maps and institutional affiliations. 
(c) (i) Open Access This article is licensed under a Creative Commons Attribution 4.0 International License, which permits use, sharing, adaptation, distribution and reproduction in any medium or format, as long as you give appropriate credit to the original author(s) and the source, provide a link to the Creative Commons license, and indicate if changes were made. The images or other third party material in this article are included in the article's Creative Commons license, unless indicated otherwise in a credit line to the material. If material is not included in the article's Creative Commons license and your intended use is not permitted by statutory regulation or exceeds the permitted use, you will need to obtain permission directly from the copyright holder. To view a copy of this license, visit http://creativecommons.org/ licenses/by/4.0/.

(C) The Author(s) 2020 